\title{
Measurement of the Dalitz-decay branching ratio of the $\pi^{0}$
}

\author{
A. Beddall ${ }^{\mathrm{a}}$, A. Beddall \\ Department of Engineering Physics, University of Gaziantep, Gaziantep 27310, Turkey
}

Received: 15 December 2007 / Revised version: 12 January 2008 /

Published online: 14 February 2008 - (C) Springer-Verlag / Società Italiana di Fisica 2008

\begin{abstract}
The ratio $\mathcal{B}=\Gamma\left(\pi^{0} \rightarrow e^{+} e^{-} \gamma\right) / \Gamma\left(\pi^{0} \rightarrow \gamma \gamma\right)$ is measured by comparing the production rates of the $\pi^{0}$ in the two decay channels. The analysis is based on approximately four million hadronic decays of the $Z$ boson recorded by the ALEPH detector in the years 1991 to 1995 . The value of $\mathcal{B}$ is determined to be $(1.140 \pm 0.041) \%$. A recalculation of the Particle Data Group world average gives rise to a slightly improved error, and moves the world average closer to the theoretical value.
\end{abstract}

\section{Introduction}

The dominant decay of the $\pi^{0}$ is to two photons with a branching ratio $\Gamma\left(\pi^{0} \rightarrow \gamma \gamma\right) \approx 98.8 \%$. Except for very rare decay modes, the remaining $1.2 \%$ follow the Dalitzdecay mode $\pi^{0} \rightarrow e^{+} e^{-} \gamma$. This decay mode was first pointed out by Dalitz [1] in 1951 where he suggested that one of the photons could internally convert into an $e^{+} e^{-}$ pair (a Dalitz pair). The Dalitz-decay has been measured in $\pi^{-} p \rightarrow \pi^{0} n$ scattering experiments by Budagov [2], Samios [3], and Schardt [4]. From these three measurements, the Particle Data Group world average for the ratio $\mathcal{B}=\Gamma\left(\pi^{0} \rightarrow e^{+} e^{-} \gamma\right) / \Gamma\left(\pi^{0} \rightarrow \gamma \gamma\right)$ is $(1.213 \pm 0.030) \%$ [5]. While this is consistent with the theoretical QED calculation of $(1.196 \pm 0.003) \%$ [5] provided by Joseph [6], the uncertainty in the ratio of about $2.5 \%$ has remained unchanged for more than a quarter of a century. An improvement in the knowledge of the Dalitz-decay branching ratio would be beneficial to branching ratio measurements of rare pion and other decays, and help in the understanding of electron backgrounds at the large hadron collider.

In this study, a new measurement of the ratio $\mathcal{B}$ is provided by studying hadronic $Z$ boson decays at the LEP collider. Such events give rise to a large number of neutral pions ( $\sim 10$ per event). Over four million hadronic events have been recorded by the ALEPH experiment over the period 1991 to 1995 . The high statistics makes it possible to observe the $\pi^{0}$ Dalitz-decay mode and measure its rate with sufficient accuracy.

In this study the Dalitz-decay rate is measured and compared with that of the latest ALEPH result for the two photon decay channel $([7])$. The ratio of the two rates therefore provides a measure of the ratio $\mathcal{B}$.

\footnotetext{
a e-mail: Andrew.Beddall@cern.ch
}

\section{The ALEPH detector}

The ALEPH detector and its performance are described in detail elsewhere $[8,9]$. Charged particles are measured over the polar angle $|\cos \theta|<0.966$ by the cylindrical inner drift chamber, and the large cylindrical time projection chamber (TPC) which measures up to 21 threedimensional space points per track. A charged-particle ionisation rate is sampled in the TPC by up to 338 wires. These chambers are immersed in a uniform magnetic field of $1.5 \mathrm{~T}$ and together measure the momentum of charged particles with a resolution $\delta_{p} / p=0.0008 p \oplus$ $0.005(p$ in $\mathrm{GeV} / c)$. For tracks with $|\cos \theta|<0.85$, which are also measured by the vertex detector, the momentum resolution is improved to $\delta_{p} / p=0.0006 p \oplus 0.005$ ( $p$ in $\mathrm{GeV} / c)$. The TPC is surrounded by the electromagnetic calorimeter (ECAL), which covers the angular range $|\cos \theta|<0.98$ and has a thickness of 22 radiation lengths. With its fine segmentation in projective towers of approximately $0.9^{\circ}$ by $0.9^{\circ}$, the angular resolution is $\sigma_{\theta, \phi}=2.5 / \sqrt{E}+0.25$ ( $E$ in $\mathrm{GeV}$ and $\sigma_{\theta, \phi}$ in mrad). This lead-proportional tube calorimeter has an energy resolution for electromagnetic showers of $\sigma_{E} / E=0.18 / \sqrt{E}+$ 0.009 ( $E$ in $\mathrm{GeV})$.

\section{Event and track selection}

Using hadronic event selection criteria defined in [10], a total of 3239746 hadronic $Z$ decays around the center-ofmass energy of $91.2 \mathrm{GeV}$ are selected for the analysis. The background to these events arises from tau pairs and twophoton events, and is estimated to be less than $0.3 \%$ [11].

Charged tracks are selected if their impact parameters are consistent with an origin close to the interac- 
tion point (transverse impact parameter $\left|d_{0}\right|<0.5 \mathrm{~cm}$ and longitudinal impact parameter $\left|z_{0}\right|<3.0 \mathrm{~cm}$ ), and if the ionisation rate is consistent with the electron hypothesis $-2<\chi_{\mathrm{d} E / \mathrm{d} x}<+4$ (where available). Here, $\chi_{\mathrm{d} E / \mathrm{d} x}=$ $\left(\mathrm{d} E /\left.\mathrm{d} x\right|_{\text {measured }}-\mathrm{d} E /\left.\mathrm{d} x\right|_{\text {expected }}\right) / \sigma_{\mathrm{d} E / \mathrm{d} x}$ where $\sigma_{\mathrm{d} E / \mathrm{d} x}$ is the expected $\mathrm{d} E / \mathrm{d} x$ resolution normalised with a sample of minimum ionising pions. Tracks are rejected if ionisation information is not available. Charged tracks are also required to have at least 4 space points in the TPC, a polar angle in the range $20^{\circ}<\theta<160^{\circ}$, and a minimum transverse momentum of $0.25 \mathrm{GeV} / c$.

The selection of photons is exactly the same as that of [7]; photons are reconstructed as neutral clusters in the ECAL and selected if their energy $E_{\gamma}$ is at least $1 \mathrm{GeV}$. Additionally, to reduce hadronic contamination, the fraction of the energy in the first two of the three segments in depth of the ECAL should be greater than 0.8 , and the fraction of the energy in the four central towers of the shower should also be greater than 0.8 .

As a means of measuring detector acceptance, a total of 6383337 Monte-Carlo events are generated with the JETSET program [12] and passed through a full detector simulation and reconstruction program. After event selection, the number of events reduces to 4923816 . In this data sample, JETSET 7.4 is tuned to describe the ALEPH data using inclusive charged particle and event shape distributions [13].

\section{Reconstruction of the Dalitz-decay}

The Dalitz-decay is reconstructed from two oppositelycharged tracks (mostly electrons identified with $\mathrm{d} E / \mathrm{d} x$ measurements), and a reconstructed photon. The invariant mass spectra contains a Dalitz signal on top of a combinatorial background, accompanied closely by a large peak due to electron pairs originating from photon conversions. In an attempt to remove the large conversion peak, and further reduce combinatorial background, two additional cuts are applied as follows.

1) Oppositely charged electron pairs are rejected if they have an invariant mass $M\left(e^{+} e^{-}\right)$greater than $0.040 \mathrm{GeV} / c^{2}$. While one would expect the reconstructed mass of a photon conversion to be close to zero, in this case the invariant mass is calculated using extrapolations of tracks with momentum vectors measured from the closest approach to the event interaction point which is a significant distance from the true points of conversion (at least $5 \mathrm{~cm}$ ); consequently the reconstructed photon conversion gains a significant mass.

2) The above cut is supplemented with an additional cut on the photon conversion radius; if a pair of reconstructed tracks (each an oppositely signed helix in the TPC) are found to have near parallel trajectories in the transverse plane and pass close together, then this point is considered to be the point of photon conversion. Such a pair of tracks is rejected if the distance $R_{\text {conv }}$ of this conversion point is greater than $4.0 \mathrm{~cm}$. This cut further improves purity, and permits a lighter cut on $M\left(e^{+} e^{-}\right)$thus improving efficiency.

These two additional cuts reduce photon conversions to an insignificant level at the expense of only $5 \%$ of the Dalitz signal. Figure 1 shows the resultant invariant mass $M\left(e^{+} e^{-} \gamma\right)$ after photon conversion rejection. The figure shows a clear signal for the Dalitz-decay mode of the $\pi^{0}$ on top of a combinatorial background. The amplitude of the backgrounds and signals are similar in the real data and Monte-Carlo data, this is a consequence of the Monte-Carlo being tuned with respect to the total $\pi^{0}$ rate and Dalitz branching ratio (taken from [5]).

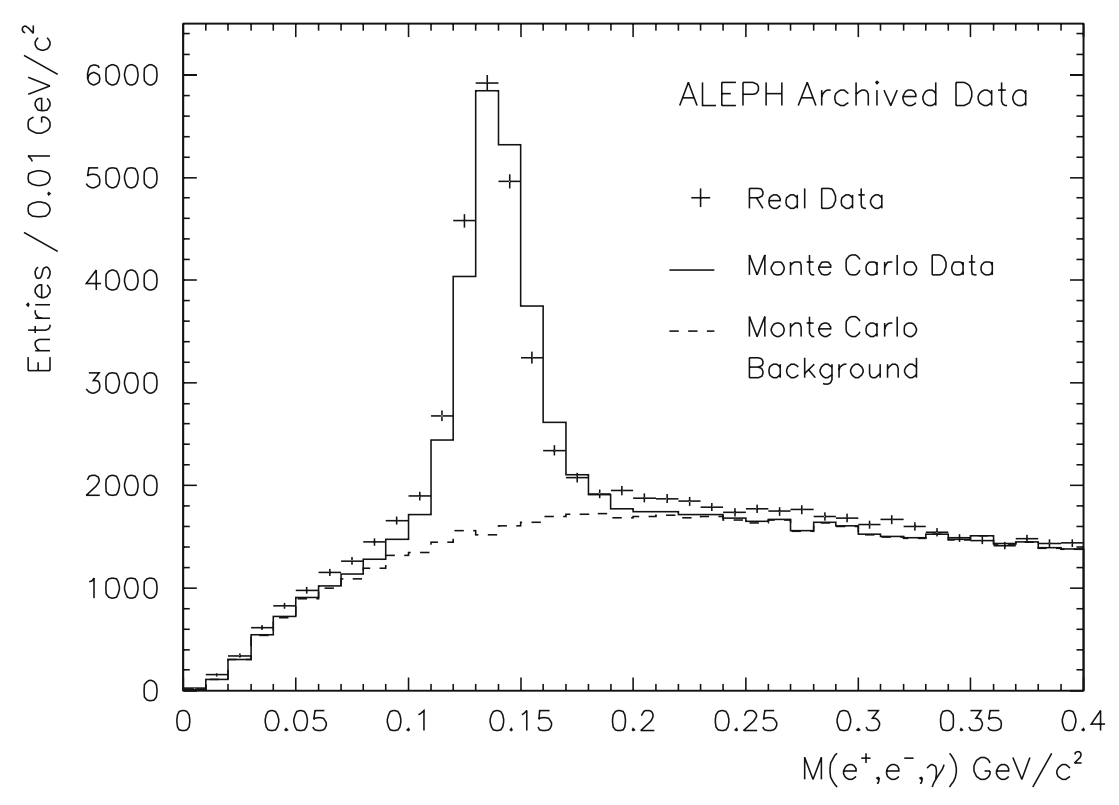

Fig. 1. Invariant mass $M\left(e^{+} e^{-} \gamma\right)$ for electron and photon candidates after rejection of photon conversions 

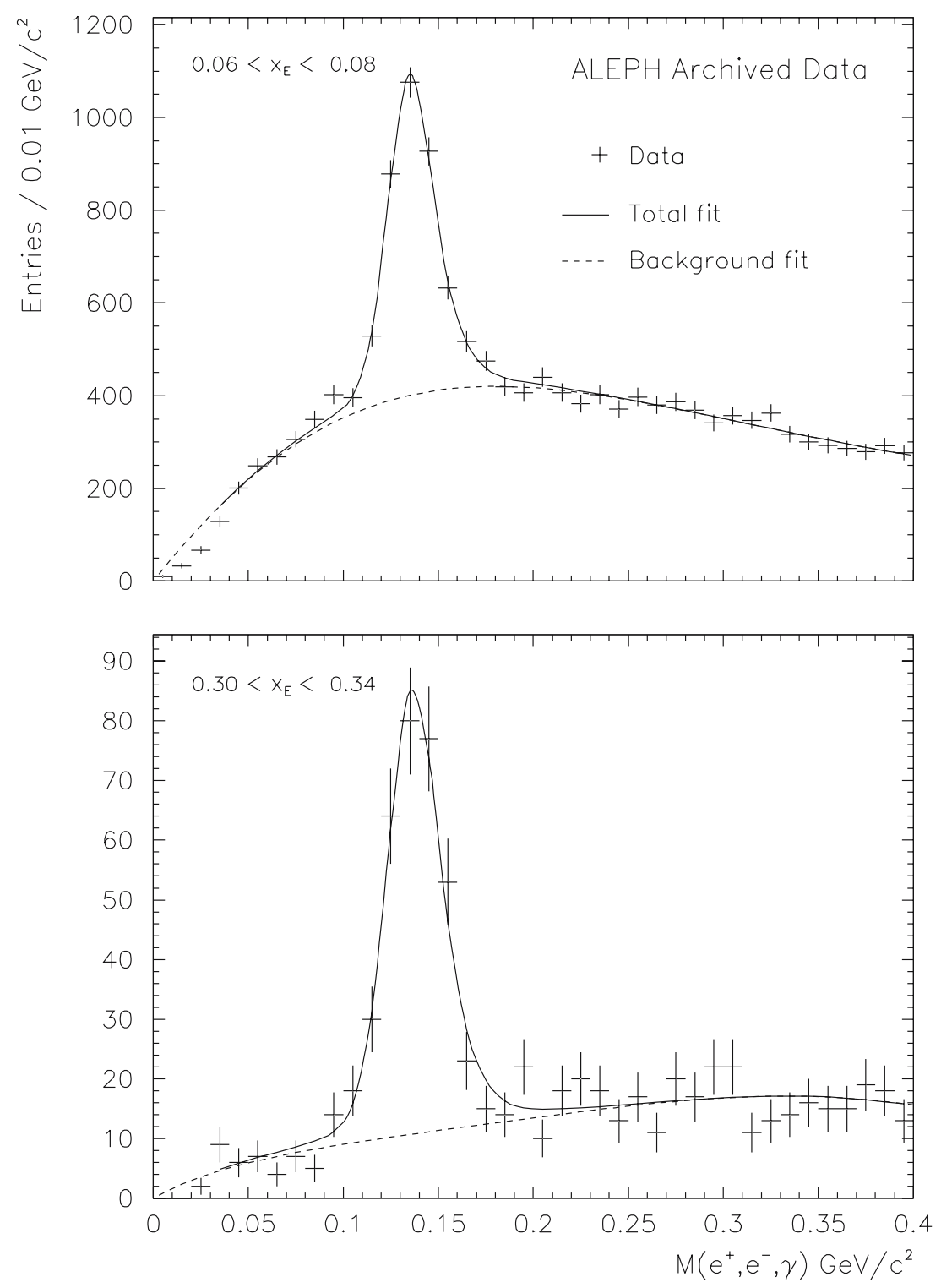

Fig. 2. Fits to invariant mass spectra in the lowest and highest measured energy intervals

\section{Extraction of the Dalitz rate}

The 'Dalitz rate' is defined as the average multiplicity, per hadronic $Z$ decay, of the $\pi^{0}$ in the decay channel $\pi^{0} \rightarrow e^{+} e^{-} \gamma$. To extract the Dalitz rate, the mass spectrum of Fig. 1 is divided in to eight intervals of scaled energy, $x_{E}=E_{\pi^{0}} / E_{\text {beam }}$ where $E_{\pi^{0}}$ is the pion energy, and $E_{\text {beam }}$ is the beam energy $=45.6 \mathrm{GeV}$. These intervals, covering the range $0.06<x_{E}<0.34$, correspond to the first eight intervals measured in [7], the remaining five intervals are omitted from this study due to their small signal significance. Each mass spectra is fitted with the sum of a signal and background function. The signal function is taken as the sum of three Gaussian functions with the relative widths, heights and peak positions optimised and fixed as constants. The global width, height, and peak position of the signal function are free parameters in the fits. The background function is chosen to be of the form $a_{0} x \exp \left(a_{1} x+a_{2} x^{2}+a_{3} x^{3}\right)$ with all parameters $a_{0}$ to $a_{3}$ allowed to be free in the fits. The total number of observed Dalitz decays in all eight measured energy intervals, estimated from the fits to the real data, is $12490 \pm 190$. Figure 2 shows the resultant fits to real data for the lowest and the highest $x_{E}$ intervals.

For each energy interval, the Dalitz rate $R_{\text {Dalitz }}$ is calculated as follows:

$$
R_{\text {Dalitz }}=\frac{S_{\mathrm{RD}} / N_{\mathrm{RD}}}{S_{\mathrm{MC}} / N_{\mathrm{MC}}} S_{\text {gen }} / N_{\text {gen }},
$$

where $S_{\mathrm{RD}}$ and $S_{\mathrm{MC}}$ are the number of signal extracted from the fits in the real data and Monte-Carlo data respectively, $N_{\mathrm{RD}}$ and $N_{\mathrm{MC}}$ are the number of events (after event selection) in the real data and Monte-Carlo data respectively, $S_{\text {gen }}$ is the number of signal in the Monte-Carlo at the generator level (before event selection), and $N_{\text {gen }}$ is the number of Monte-Carlo events (before event selection). 


\section{Statistical and systematic errors}

The statistical errors on the measured value of $R_{\text {Dalitz }}$ are taken from the statistical uncertainty in the fits to the signal in the real data and Monte-Carlo data, i.e the statistical errors in the values of $S_{\mathrm{RD}}$ and $S_{\mathrm{MC}}$ respectively.

With respect to systematic errors, this study relies on the correct simulation of the reconstruction of both charged tracks (dominated by the the TPC), and electromagnetic clusters (reconstructed in the ECAL). However, systematics in photon reconstruction are considered to be dominant and so no detailed studies of tracking systematics are performed. While this study (and the study in [7] for the two-photon channel) takes advantage of the improvements gained by the final re-processing of the ALEPH data, some well known problems still remain with respect to the simulated acceptance of low energy photons, photon energy calibration, and ECAL energy clusters originating from hadrons or other sources of contamination such as satellite clusters. To minimise these effects, cuts are placed on $E_{\gamma}$ and on energy fractions in ECAL towers as discussed in Sect. 3. Systematics relating to the above effects are considered and estimated in [7]. As these systematics are common to both studies, the error estimates taken from [7] are halved to take into account partial systematics cancellation due to correlations between the two measurements. Furthermore the systematic errors are treated in [7] as correlated between energy intervals and so are summed linearly, this conservative approach is preserved in this study.

Other contributions to systematic errors are also considered. They include the statistical uncertainty in the generator level signal $S_{\text {gen }}$, variations in the measured rate due to different choices of the fitting range and the values of key cuts. The latter contributions are determined by varying, within a reasonable range, the values of cuts as follows. The cut on the transverse impact parameter $\left|d_{0}\right|$ is reduced to $0.3 \mathrm{~cm}$ and increased to $1.0 \mathrm{~cm}$, the lower value of $\chi_{\mathrm{d} E / \mathrm{d} x}$ is decreased to -3.0 and increased to -1.5 , the minimum value of $E_{\gamma}$ is decreased to $0.8 \mathrm{GeV}$ and increased to $1.2 \mathrm{GeV}$, the cut on $M\left(e^{+} e^{-}\right)$is decreased to $0.030 \mathrm{GeV} / c^{2}$ and increased to $0.050 \mathrm{GeV} / c^{2}$, and the cut on $R_{\text {conv }}$ is decreased to $3.0 \mathrm{~cm}$ and increased to $5.0 \mathrm{~cm}$. The maximum variation in the rate for each cut variation is assigned as a systematic error.

These results, together with the statistical errors, are shown in Table 1. It should be noted that, for all the cut variations, the sign of the variations in the rates between each energy interval is seen to be random. The errors are therefore considered to be uncorrelated and so are summed in quadrature over energy intervals.

\section{Results}

Table 2 shows the measured rates for the two $\pi^{0}$ decay channels. The Dalitz rate $R_{\text {Dalitz }}$ is measured in the present study, and the two-photon rate $R_{\gamma \gamma}$ is taken from the study of [7]. The ratio $\mathcal{B}$ is calculated by dividing the two rates as follows.

$$
\mathcal{B}=\Gamma\left(\pi^{0} \rightarrow e^{+} e^{-} \gamma\right) / \Gamma\left(\pi^{0} \rightarrow \gamma \gamma\right)=R_{\text {Dalitz }} / R_{\gamma \gamma}
$$

Column 'All' in the table represents the weighted sum of the results over all energy intervals. For this, the summations are performed in quadrature except for the systematic error of the two-photon rate which is summed linearly. As a further systematic check, the value of $\mathcal{B}$ is compared for each $x_{E}$ interval, this is illustrated in Fig. 3. From this figure, it can be seen that all values are consistent, and do not appear to be correlated with energy. The ALEPH result for the ratio $\mathcal{B}$ is compared to the results of previous studies [5] in Table 3 . The values of $n_{\sigma}$ in the table show the number of standard deviations the measured results are from the theoretical value. These results are also illustrated in Fig. 4.

Table 1. Systematic and statistical errors for the measured $\pi^{0}$ rates in each measured $x_{E}$ interval. Errors for the Dalitz-decay channel $\pi^{0} \rightarrow e^{+} e^{-} \gamma$ are taken from the present study, and errors for the decay $\pi^{0} \rightarrow \gamma \gamma$ are taken from [7]. All values are expressed in percent rounded to two decimal places

\begin{tabular}{lccccccccc}
\hline$\pi^{0} \rightarrow e^{+} e^{-} \gamma$ & & \multicolumn{9}{c}{ Measured $x_{E}$ interval } & & \\
Error source & All & 1 & 2 & 3 & 4 & 5 & 6 & 7 & 8 \\
\hline$d_{0}$ & 0.59 & 1.06 & 1.44 & 1.40 & 1.72 & 1.91 & 2.20 & 0.91 & 1.18 \\
$\chi_{\mathrm{d} E / \mathrm{d} x}$ & 1.40 & 4.58 & 0.88 & 1.31 & 3.02 & 0.57 & 2.63 & 5.74 & 8.26 \\
$E_{\gamma}$ & 0.96 & 2.27 & 2.93 & 1.01 & 2.45 & 0.26 & 0.35 & 1.67 & 0.38 \\
$\left.M^{+} e^{+} e^{-}\right)$ & 1.04 & 1.37 & 3.01 & 2.62 & 1.23 & 5.01 & 1.89 & 3.80 & 6.36 \\
$R_{\text {conv }}$ & 0.49 & 0.88 & 0.72 & 1.47 & 1.60 & 0.61 & 0.57 & 1.54 & 2.18 \\
$S_{\text {gen }}$ & 0.25 & 0.45 & 0.56 & 0.52 & 0.70 & 0.91 & 1.15 & 1.45 & 1.72 \\
Fit range & 0.96 & 1.64 & 2.79 & 1.46 & 3.06 & 2.40 & 5.91 & 2.20 & +6.64 \\
$\pi^{0} \rightarrow e^{+} e^{-} \gamma$ & & & & & & & & & \\
Systematic error & 2.35 & 5.72 & 5.40 & 4.01 & 5.66 & 6.01 & 7.21 & 7.77 & 12.73 \\
Statistical error & 2.09 & 5.15 & 4.83 & 3.62 & 4.50 & 5.35 & 7.00 & 7.80 & 10.05 \\
$\pi^{0} \rightarrow \gamma \gamma([7])$ & & & & & & & & & \\
Systematic error & 1.77 & 1.61 & 1.65 & 1.80 & 2.05 & 2.21 & 1.89 & 1.68 & 1.45 \\
Statistical error & 0.30 & 0.81 & 0.65 & 0.43 & 0.46 & 0.50 & 0.58 & 0.93 & 1.29 \\
\hline
\end{tabular}


Table 2. Measured $\pi^{0}$ rates in two decay channels. The results for the Dalitz-decay channel $\pi^{0} \rightarrow e^{+} e^{-} \gamma$ are taken from the present study, and results for the decay $\pi^{0} \rightarrow \gamma \gamma$ are taken from [7]

\begin{tabular}{lccccccccc}
\hline Measured $x_{E}$ interval & All & 1 & 2 & 3 & 4 & 5 & 6 & 7 \\
\hline Dalitz rate, $R_{\text {Dalitz }}$ & 0.02347 & 0.00652 & 0.00492 & 0.00523 & 0.00295 & 0.00176 & 0.00098 & 0.00066 & 0.00046 \\
Statistical error & 0.00049 & 0.00034 & 0.00024 & 0.00019 & 0.00013 & 0.00009 & 0.00007 & 0.00005 & 0.00005 \\
Systematic error & 0.00055 & 0.00037 & 0.00027 & 0.00021 & 0.00017 & 0.00011 & 0.00007 & 0.00005 & 0.00006 \\
Two-photon rate, $R_{\gamma \gamma}$ & 2.05984 & 0.61640 & 0.40040 & 0.46600 & 0.24988 & 0.14452 & 0.08972 & 0.05580 & 0.03712 \\
Statistical error & 0.00620 & 0.00500 & 0.00260 & 0.00200 & 0.00116 & 0.00072 & 0.00052 & 0.00052 & 0.00048 \\
Systematic error & 0.03640 & 0.00990 & 0.00660 & 0.00840 & 0.00512 & 0.00320 & 0.00170 & 0.00094 & 0.00054 \\
$\mathcal{B}=R_{\text {Dalitz }} / R_{\gamma \gamma}$ & 1.13952 & 1.05772 & 1.22758 & 1.12161 & 1.17862 & 1.21746 & 1.09285 & 1.18787 & 1.24635 \\
Statistical error & 0.02408 & 0.05516 & 0.05985 & 0.04088 & 0.05337 & 0.06546 & 0.07679 & 0.09328 & 0.12628 \\
Systematic error & 0.03349 & 0.06287 & 0.06929 & 0.04935 & 0.07097 & 0.07795 & 0.08150 & 0.09441 & 0.15966 \\
Total Error & 0.04125 & 0.08364 & 0.09156 & 0.06408 & 0.08880 & 0.10179 & 0.11197 & 0.13272 & 0.20357 \\
\hline
\end{tabular}
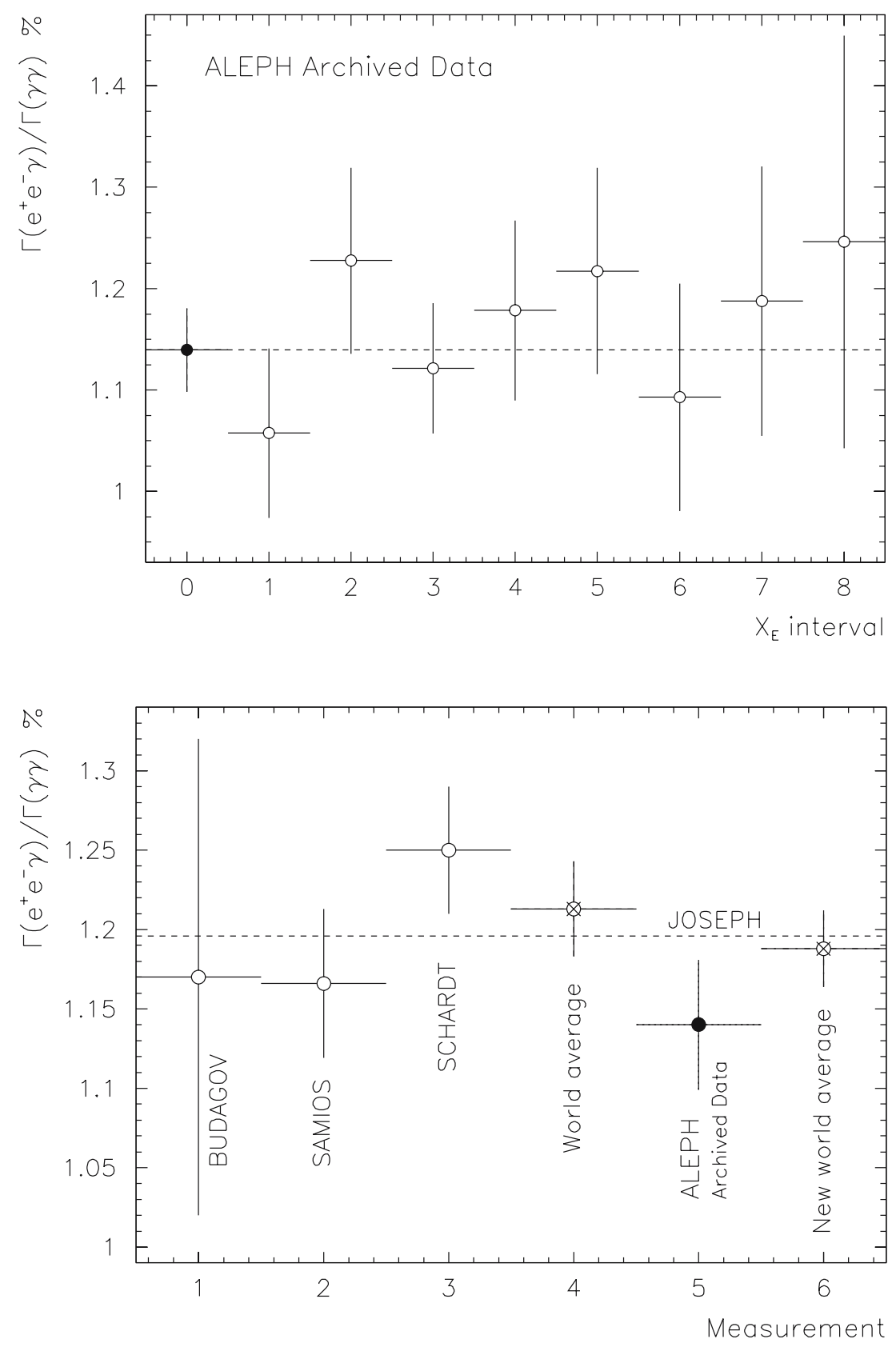

Fig. 3. The ratio $\mathcal{B}$ for the eight measured $x_{E}$ intervals. The first point (closed circle), and the dashed line, represents the ratio for the total measured interval (column 'All' in Table 2)

Fig. 4. Values for the ratio $\mathcal{B}$ from each study. The error bars are the quadrature sum of the statistical and systematic contributions 
Table 3. Values for the ratio $\mathcal{B}$ from each study. The first value is from the theoretical QED calculation of Joseph

\begin{tabular}{lcc}
\hline Study & $\mathcal{B}=\Gamma\left(e^{+} e^{-} \gamma\right) / \Gamma(2 \gamma)$ & $n_{\sigma}$ \\
\hline Joseph 1960 & $(1.196 \pm 0.003) \%$ & theory \\
Budagov 1960 & $(1.170 \pm 0.150) \%$ & -0.2 \\
Samios 1961 & $(1.166 \pm 0.047) \%$ & -0.6 \\
Schardt 1981 & $(1.250 \pm 0.040) \%$ & +1.4 \\
ALEPH* 2008 & $(1.140 \pm 0.041) \%$ & -1.4 \\
Weighted average: & & \\
excluding ALEPH & $(1.213 \pm 0.030) \%$ & +0.6 \\
including ALEPH & $(1.188 \pm 0.024) \%$ & -0.3 \\
\hline
\end{tabular}

* ALEPH Archived Data

\section{Summary and discussion}

Hadronic decays of the $Z$ boson give rise to a large number of neutral pions. Over four million such events have been recorded by the ALEPH experiment over the period 1991 to 1995. The high statistics makes it possible to observe the Dalitz-decay of the $\pi^{0}$ with sufficient signal significance to measure its rate. Using the same data, [7] provides a measurement of the two-photon decay channel of the $\pi^{0}$. The ratio of these two measurements therefore leads to a measurement of the ratio $\mathcal{B}=\Gamma\left(\pi^{0} \rightarrow e^{+} e^{-} \gamma\right) / \Gamma\left(\pi^{0} \rightarrow\right.$ $\gamma \gamma)$ which has not been measured since 1981. This approach takes advantage of the partial cancellation of some systematic errors as a component of both measurements shares the same type of uncertainty.

The previous measurements for this ratio, used by the particle data group [5] to form a world average, and the result of the present study, are given in Table 3 and illustrated in Fig. 4. While the result from this study does not have a greater accuracy than that of Samios and Schardt, it provides additional statistical information reducing the uncertainty in the world average from about $2.5 \%$ to $2.0 \%$. The new average also moves closer, from $+0.6 \sigma$ to $-0.3 \sigma$, to the theoretical prediction.
With similar capablilities, other LEP experiments (DELPHI, L3 and OPAL) could measure the Dalitz-decay with a comparable accuracy. If so, such a combined effort may result in a measurment of the Dalitz decay branching ratio with a uncertainty well below $2 \%$.

Acknowledgements. We wish to thank the ALEPH collaboration for access to the archived data since the closure of the collaboration [14], and the ALEPH editorial board for their time and comments. We also wish to thank the CERN accelerator divisions for the successful operation of LEP, and we are indebted to the researchers, engineers and technicians for their contribution to the excellent performance of ALEPH.

\section{References}

1. R.H. Dalitz, Proc. Phys. Soc. (London) 64, 667 (1951)

2. Y.A. Budagov et al., Sov. Phys. JETP 11, 755 (1960) (translated from Zh. Eksp. Teor. Fiz. 38, 1047 (1960))

3. N.P. Samios, Phys. Rev. 121, 275 (1961)

4. M.A. Schardt et al., Phys. Rev. D 23, 639 (1981)

5. Particle Data Group, W.M. Yao et al., J. Phys. G 33, 1 (2006)

6. D.W. Joseph, Nuovo Cim. 16, 997 (1960)

7. ALEPH Collaboration, R. Barate et al., Eur. Phys. J. C 16, 613 (2000)

8. ALEPH Collaboration, D. Decamp et al., Nucl. Instrum. Methods A 294, 121 (1990)

9. ALEPH Collaboration, D. Buskulic et al., Nucl. Instrum. Methods A 360, 481 (1995)

10. ALEPH Collaboration, D. Buskulic et al., Z. Phys. C 69, 379 (1996)

11. ALEPH Collaboration, D. Decamp et al., Z. Phys. C 53, 1 (1992)

12. T. Sjöstrand, Comput. Phys. Commun. 82, 74 (1994)

13. ALEPH Collaboration, R. Barate et al., Phys. Rep. 294, 1 (1998)

14. Statement on use of ALEPH data for long-term analyses (http://aleph.web.cern.ch/aleph/alpub/archive_data.pdf) 